\title{
Endothelial tip cell formation induced by chronic lymphocytic leukemia plasma (JAK2 positivity amplified this effect)
}

\author{
Minoo Shahidi ${ }^{1,2} \cdot$ Najibeh Esmaeili $^{2} \cdot$ Mohammad Faranoosh $^{3} \cdot$ Ahmad Kazemi $^{2}$ • \\ Mahmood Barati ${ }^{4} \cdot$ Nafise Ansarinejad $^{3}$
}

Received: 24 November 2016/Accepted: 12 April 2017 /Published online: 20 April 2017

(C) Springer-Verlag Berlin Heidelberg 2017

\begin{abstract}
A subset of specialized endothelial cells, called tip cells, which guide expanding capillaries toward gradients of vascular endothelial growth factor (VEGF), are responsible for growing of capillaries during angiogenesis. Certain evidence has suggested the involvement of angiogenesis in the pathophysiology of chronic lymphocytic leukemia. Nevertheless, the direct effect of chronic lymphocytic leukemia (CLL) plasma on tip cell formation is yet to be fully determined. We aimed to evaluate whether the endothelial cells are able to form tip cells in the presence of CLL plasma. Human umbilical vein endothelial cells were treated with CLL patient's plasmas. The expression of CD34 and vascular endothelial growth factor receptor-2 (VEGFR2) levels were detected by flow cytometry, qPCR, and immunocytochemical staining techniques. We found that CD34- and VEGFR2positive cells were increased following direct exposure to CLL plasma. Nevertheless, the presence of a higher absolute lymphocyte count and/or Janus kinase (JAK2) mutation could amplify both CD34 and VEGFR2 expression in cell culture. Our findings may suggest CLL plasma as a potential source of growth factors, which might be responsible for tip cell
\end{abstract}

Minoo Shahidi

shahidi.minoo@gmail.com; Shahidi.m@iums.ac.ir

1 Cellular and Molecular Research Center (CMRC), Iran University of Medical Sciences, Tehran, Iran

2 Hematology and blood banking departments, Faculty of Allied Medicine, Iran University of Medical Sciences, Hemmat Highway, Tehran 14155-598, Iran

3 Hazrat Rasul Medical complex, Iran University of Medical Sciences, Tehran, Iran

4 Department of Biotechnology, Faculty of Allied Medicine, Iran University of Medical Sciences, Tehran, Iran development. Nevertheless, the JAK2 mutation may potentiate this effect.

Keywords Chronic lymphocytic leukemia $\cdot$ Human umbilical vein endothelial cells, tip cells $\cdot$ Cell culture

\section{Introduction}

Tip cells as the principal cells in sprouting angiogenesis could sense their environment for guidance signals $[1,2]$. Accordingly, these cells have been known as promising therapeutic target for antiangiogenic therapies, particularly in malignant disorders including leukemia [3, 4]. Studies, however, have characterized tip cells with filopodia in human umbilical vein endothelial cell (HUVEC) cultures, which could be recognized by overexpression of CD34, as an in vitro model for the study of tip cell biology [5, 6]. Nevertheless, the newly generated sprouts are guided by these migrating endothelial tip cells, which are sensitive to signal transduction from the extracellular microenvironment [1]. In this respect, intracellular signaling through vascular endothelial growth factor receptors-2 (VEGFR2), which is highly enriched in tip cells, promotes angiogenesis [6]. Subsequently, other endothelial cells could follow the migrating tip cell to produce vascular lumen $[4,7]$. In this regard, interaction of chronic lymphocytic leukemia (CLL) plasma, containing exosomes together with angiogenic factors, with endothelial cells as the key cells in the angiogenic process might be operative. It has been established that in chronic lymphocytic leukemia, upon infiltration of the BM with leukemia cells, angiogenesis could be developed [8]. Although leukemic cell interactions with microenvironment have been considered as a critical issue for development of CLL [9], there is no in vitro study to investigate the CLL plasma effects on endothelial cell alteration to tip cell. 
Previously, we found a proliferative effect of the CLL plasma together with vWF increased level in endothelial cell culture [10]. Accordingly, evaluation of cells overexpressing CD34 and VEGFR2, which are linked with angiogenesis and migration, seems to be valuable. Thus, the present study aimed to detect endothelial tip cell development after treatment with CLL plasma.

\section{Methods}

\section{Cells}

Recent studies have established the human umbilical vein endothelial cells (HUVECs) as an in vitro model for study of tip cell biology [5]. Therefore, HUVECs were obtained from the National Cell Bank of Iran (NCBI) affiliated to Pasteur Institute of Iran. HUVECs were maintained in complete medium: Dulbecco's modified Eagles medium (DMEM, Gibco), $10 \%$ heat-inactivated fetal bovine serum (FBS, Gibco, Invitrogen, UK), $50 \mathrm{U} / \mathrm{ml}$ penicillin $\mathrm{G}$, sodium salt, and $50 \mu \mathrm{g} / \mathrm{ml}$ streptomycin sulfate (P/S, Gibco). The cells were maintained in a $37^{\circ} \mathrm{C}$ incubator with $5 \% \mathrm{CO}_{2}$.

\section{Sample preparation}

Blood samples were collected from 20 CLL patients (before treatment) admitted to the hematology unit of the Hazrat Rasoul Hospital (Iran University of Medical Sciences) between November, 2014 and October, 2015, and 17 patients were included in this study. The samples were also collected from normal subjects within the same range of age, and the hematological parameters were examined for all. Immunophenotyping of the leukemic cells was carried out using antibodies against CD3, CD5, CD19, CD22, CD23, and HLADR (Sigma Aldrich, UK). All of the cases were found as B-cell CLL. Besides, detection of Janus kinase (JAK2) mutation revealed one positive case among the subjects as was carried out using PCR.

\section{MTT assay}

Cells were plated in 96-well plates in DMEM (2\% FBS) at a concentration of $5 \times 10^{3}$ cells $/ \mathrm{ml}$ and incubated for $4 \mathrm{~h}$ at $37^{\circ} \mathrm{C}$ prior to treatment. The cells were treated with plasma and each dose was plated in triplicate. Following the addition of $100 \mu \mathrm{l} / \mathrm{well}$ thiazolyl blue tetrazolium bromide (MTT) solution ( $5 \mathrm{mg} / \mathrm{ml}$; Sigma Aldrich), the plate was incubated at $37{ }^{\circ} \mathrm{C}$ until an appropriate purple coloration was settled. The optical density of the untreated controls was taken as $100 \%$ cell viability, and all subsequent readings were calculated as a percentage of the control.

\section{Real-time polymerase chain reaction}

To support the effect of CLL plasma on CD34 and VEGFR2 expression, real-time polymerase chain reaction (PCR) was carried out to evaluate the tip cell formation. HUVECs were cultured in medium flasks $\left(3 \times 10^{5}\right.$ cells/ $\mathrm{ml}$ ) with B-cell CLL plasma, normal plasma, and medium alone 3 days before treatment. The cells were harvested after $12-48 \mathrm{~h}$ and washed with PBS. Total RNA was extracted from the cells (AccuZol ${ }^{\mathrm{TM}}$, Bioneer) subsequently. The reverse transcription reaction was conducted (using $1 \mu \mathrm{g}$ of RNA) using the cDNA synthesis kit (Bioneer, AccuPower Cycle Script RT PreMix) following the manufacturer's instructions. The expression levels of mRNA encoding human CD34, VEGFR2, and Sp1 (as the housekeeping genes) were quantified with a Rotor-Gene-6000 (Corbett Research, Sydney, Australia), using the $\Delta \Delta \mathrm{CT}$ Method. The PCR reaction was performed in $10 \mu \mathrm{l}$ volume containing $5 \mu \mathrm{l}$ PCR Master Mix (AccuPower 2X Greenstar qPCR Master Mix, Bioneer), $1 \mu \mathrm{l}$ cDNA (50 ng), $2 \mu \mathrm{l}$ PCR-grade water, and $10 \mathrm{pmol}$ of specific primers (Invitrogen, UK) for CD34 (forward 5'-TTGA CAACAACGGTACTGCTAC-3' and reverse 5'-TGGT GAACACTGTGCTGATTAC-3'), VEGFR2 (forward 5' ATTCCTCCCCCGCATCA $3^{\prime}$ and reverse $5^{\prime}$ GCTCGTTGGCGCACTCTT 3') and Sp1 (forward 5' TCGGATGAGCTACAGAGGCACAAA and reverse 5' AAAGTGCCCACACTCAGAGCTACA). Thermal cycling conditions were as follows: $95{ }^{\circ} \mathrm{C}$ for $5 \mathrm{~min}, 40 \mathrm{cy}-$ cles at $95{ }^{\circ} \mathrm{C}$ for $15 \mathrm{~s}, 60{ }^{\circ} \mathrm{C}$ for $45 \mathrm{~s}$, and finally a melting curve program from 60 to $95{ }^{\circ} \mathrm{C}$. All assays were carried by triplicate. The mRNA abundance was calculated according to the comparative threshold cycle $(\mathrm{Ct})$ method, where the amount of targets (CD34, VEGFR2), normalized to the endogenous reference (Sp1) and relative to the control, is given by $2^{-\Delta \Delta \mathrm{Ct}}$.

\section{NF-kB inhibition}

To inhibit the NF-kB transcription factor by small interfering RNA (siRNA) against NF-kB (on-target plus siRNA no. 1 NF-kB Human Dharmacon), 1 day before transfection, the cells were cultured in 24-well plates at $5 \times 10^{4}$ cells $/ \mathrm{ml}$ in growth medium with reduced FBS (2\%) concentration without antibiotics to reach $70 \%$ confluence at the time of transfection. The transfection was carried out as described previously [11]. Thereafter, transfected cells were challenged with normal and CLL plasma for an extra $24 \mathrm{~h}$ at $37^{\circ} \mathrm{C}$ in a humidified $\mathrm{CO} 2$ incubator. 
Fig. 1 The result of the MTT test. Cells were plated and treated with various concentrations of plasma; each dose was plated in triplicate. The top line shows viability and the bottom line shows the plasma concentration

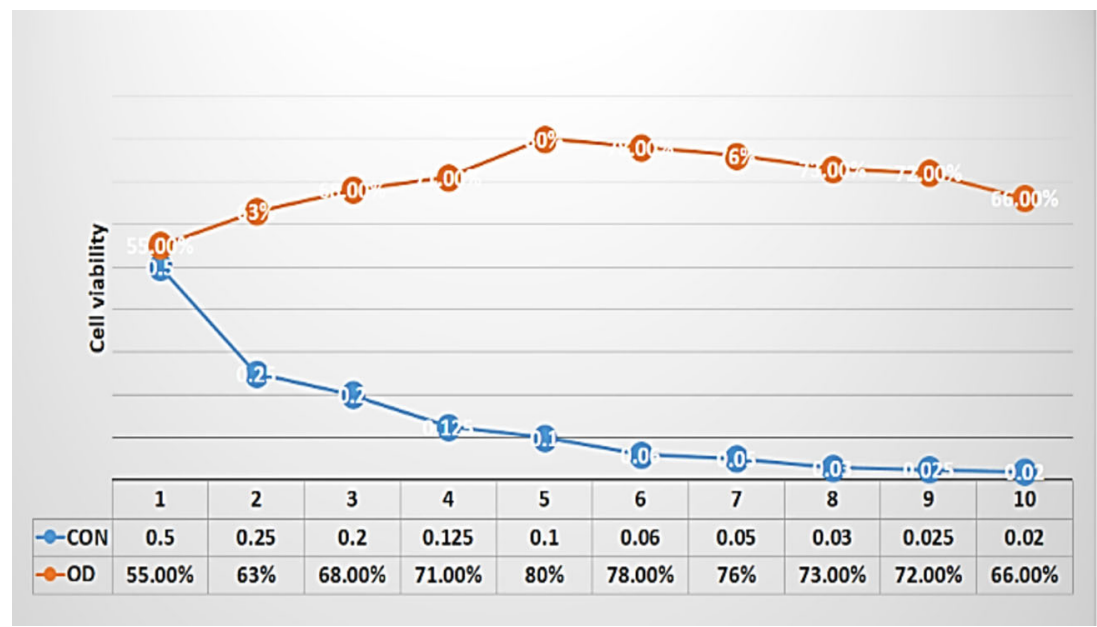

\section{Immunophenotyping}

The cells were seeded and experiments were performed over 3 days (using the cells between passages 5 and 8). For starvation of the cells, they were cultured with reduced FBS (2\%) concentration and were treated for $12-48 \mathrm{~h}$ with plasma $1 / 10$ as optimized with the MTT test for $24-48 \mathrm{~h}$. Then, the cells were harvested and immunofluorescent labeling and washing were performed with PBS containing $0.1 \%$ bovine serum albumin. Next, the cells were fixed in $2 \%$ paraformaldehyde in PBS for $15 \mathrm{~min}$ at RT and incubated with anti-CD34 (antiCD34-FITC; Dako).

\section{Immunocytochemical staining}

The HUVECs were cultured by adding $25 \mathrm{ml}$ of cultured media containing 50,000 cells to the four-well plate with $2 \%$
FBS and $1 \%$ penicillin-streptomycin. Cells were incubated at $37{ }^{\circ} \mathrm{C}$ and $5 \% \mathrm{CO} 2$ in air. When the desired density was reached, the cells were treated with normal and CLL patients' plasmas. The cells were then incubated for $24 \mathrm{~h}$ at $37{ }^{\circ} \mathrm{C}$ and $5 \% \mathrm{CO} 2$ in air. The cells were washed twice using phosphate-buffered saline (PBS). Four hundred milliliters of $4 \%$ paraformaldehyde in PBS was added to each well and incubated for $20 \mathrm{~min}$ at RT. The cells were washed twice with PBS and covered with $400 \mathrm{ml}$ of wash buffer. To permeabilize the cells, they were incubated in $0.1 \%$ Triton X-100 in PBS for $15 \mathrm{~min}$ at RT. Then, the cells were rinsed three times with PBS and blocked with 5\% normal goat serum. Anti-VEGFR2 antibody (Abcam, UK) was added at a dilution of 1: 200 $(50 \mathrm{ml})$, and the cells were incubated at $4{ }^{\circ} \mathrm{C}$ overnight. After washing with PBS, the cells were stained
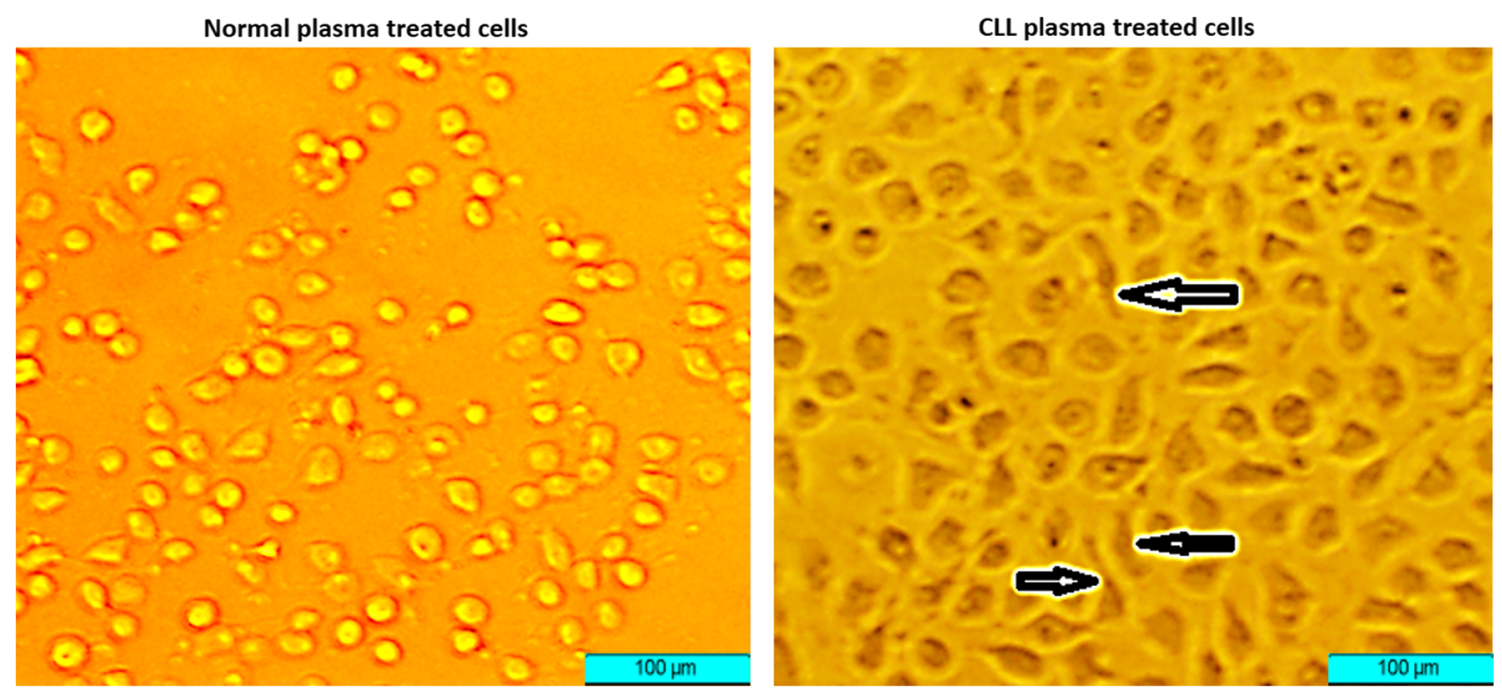

Fig. 2 The morphology alteration of the endothelial cells. Some cell morphology was altered from cobblestone to extended filopodia as notified by arrows in the figure 

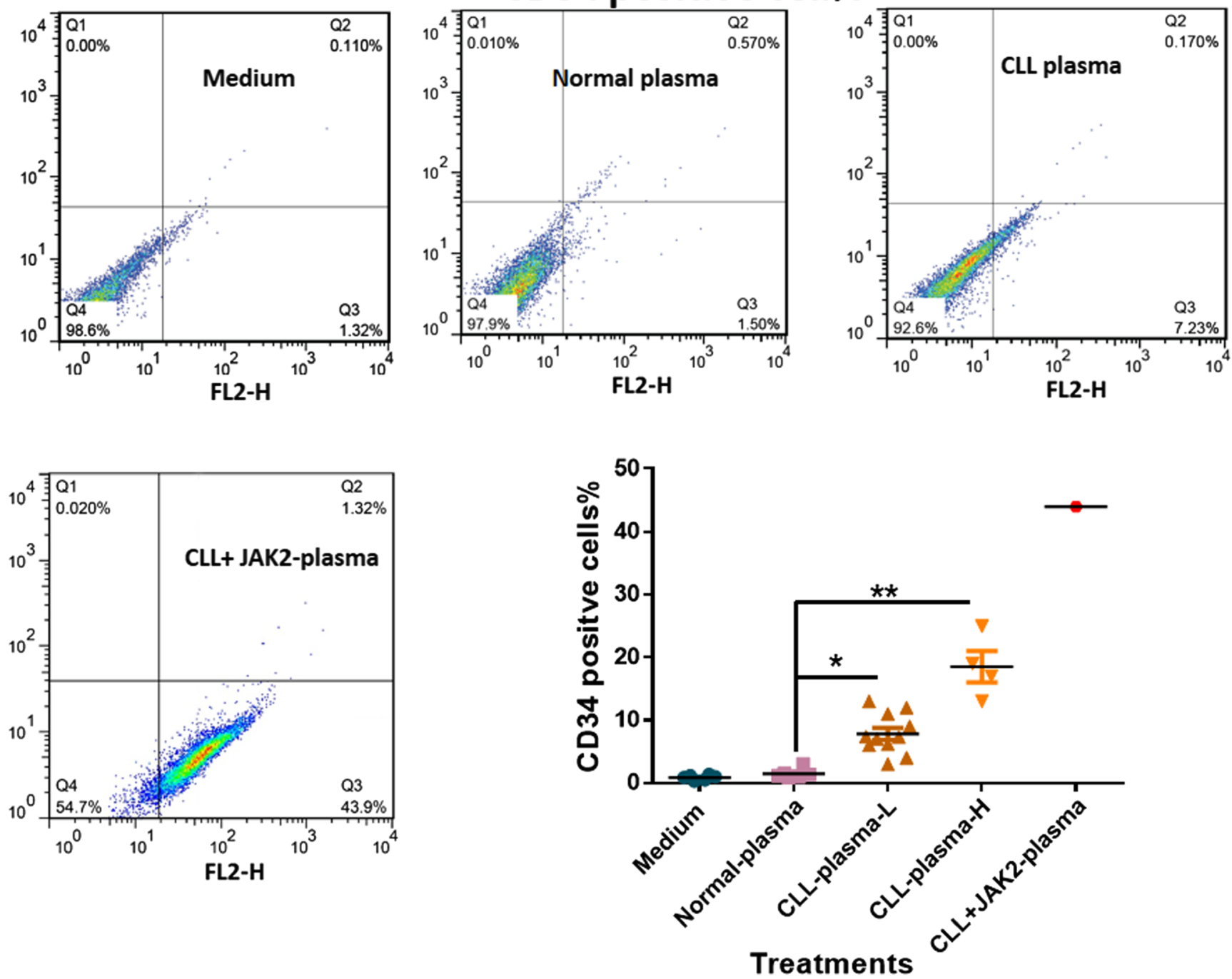

Fig. 3 The result of the tip cell detection. Top: representative flow cytometric dot plots of HUVEC after treatment with medium alone, normal, and CLL plasma. Bottom: percentage of CD34-positive cell population in different treatment. JAK2 ${ }^{+}$CLL plasma could increase CD34 positive cells dramatically as it is shown in the figure. Individual

data are presented with mean $\pm \mathrm{SD}$ ( $t$ test) using flow cytometry, where $* p<0.05$ and $* * p<0.01$ when compared to cells incubated with normal plasma. CLL-plasma-L CLL-plasma of cases with lower absolute leukemic cells, CLL-plasma-H CLL-plasma of cases with higher absolute leukemic cells, HUVEC human umbilical vein endothelial cell

with secondary antibody (thermo fisher, UK) and were incubated once more. Subsequent imaging was performed using fluorescence microscopy and analyzed via ImageJ software.

\section{Statistical analysis}

To compare the values, student's $t$ test was used. The linear correlations between the different parameters were evaluated using the Spearman's rank-sum correlation method. The findings were considered significant, when $p$ value was less than 0.05 .

\section{Results}

The results of at least three experiments for each experimental condition were analyzed. Immunophenotypic detection of leukemic cell was carried out using antibodies against CD3, CD5, CD19, CD22, CD23, and HLADR (Sigma Aldrich, UK). JAK2 mutation was reported just in one of the cases. The patient's white blood cell count was raised to $29.6 \times 10^{9} / 1$, whereas his hemoglobin and hematocrit were within the reference ranges. His platelet count, however, was decreased to $120 \times 10^{9} / 1$. The circulating lymphocyte percentage was $81 \%$, and the total lymphocyte count was $21 \times 10^{9} / 1$. The analysis of the 
Fig. 4 The result of the qPCR. The histograms demonstrate the CD34 and VEGFR2 mRNA expression after treatment with medium, normal, and CLL plasma for $48 \mathrm{~h}$. Values were obtained from three independent experiments and were normalized to Sp1 (housekeeping gene). Individual data are presented with mean $\pm \mathrm{SD}(t$ test $)$, where $* p<0.05$ and $* * p<0.01$ when compared to cells incubated with normal plasma. $C L L$ chronic lymphocytic leukemia, HUVEC human umbilical vein endothelial cell

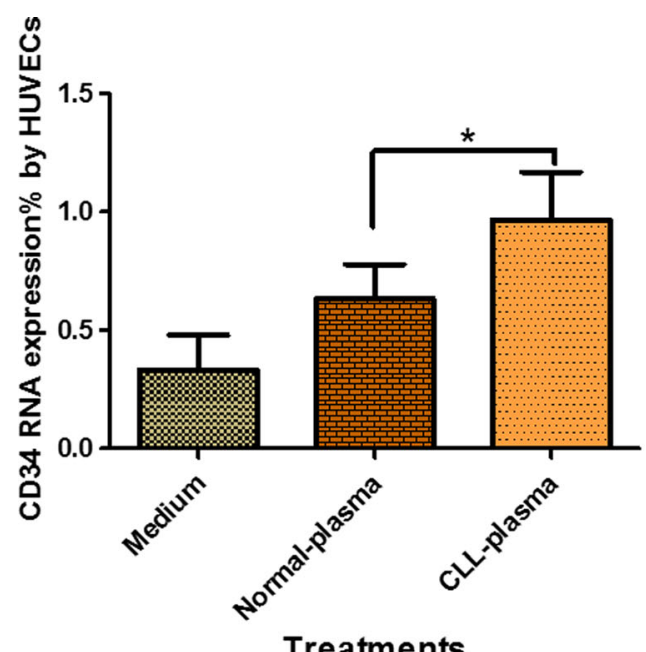

Treatments

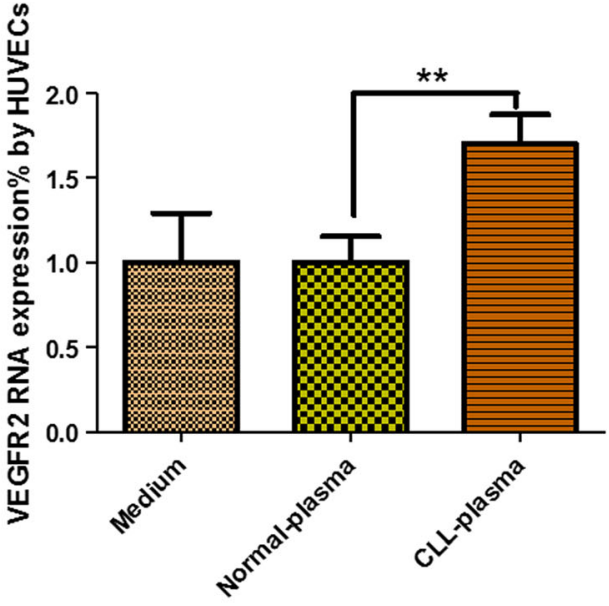

Treatments lymphocytes demonstrated B-CLL phenotype as analyzed by flow cytometry [CD19 (83.1\%), CD20 (79.7\%), CD5 $(83.9 \%)$, CD22 (52.1\%), CD3 (16.4\%) and HLA-DR $(71.8 \%)]$.

\section{MTT assay}

The cells were cultured with reduced FBS $(2 \%)$ concentration and treated over 24-48 h with both CLL plasma and normal plasma $1 / 10$ as optimized with MTT test (Fig. 1).

The effects of CLL plasma on the morphology of HUVECs were evaluated and compared with those of the normal plasma. Treatment with CLL plasma changed some cobblestone cell morphology to extended filopodia (Fig. 2).

\section{FACS sorting of the CD34-positive cells}

To elucidate endothelial alteration to tip cell, CD34 expression was detected on the surface of endothelial cells via a CD34 antibody (Abcam, UK). There was a significant increase in the number of CD34-positive cells in HUVECs culture after treatment with CLL plasma for 24-48 h using anti-CD34 antibody and flow cytometry method, when compared to the cells treated with normal plasma (control, $p=0.0043$, Fig. 3). NF-kB

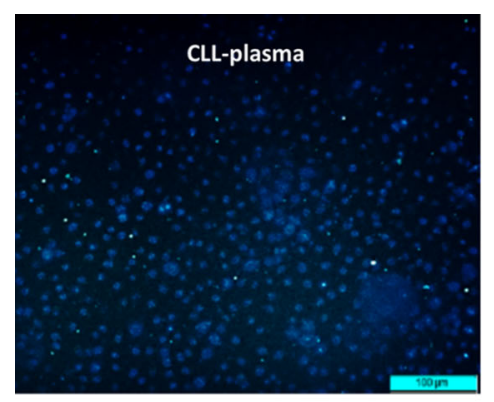

Normal-plasma
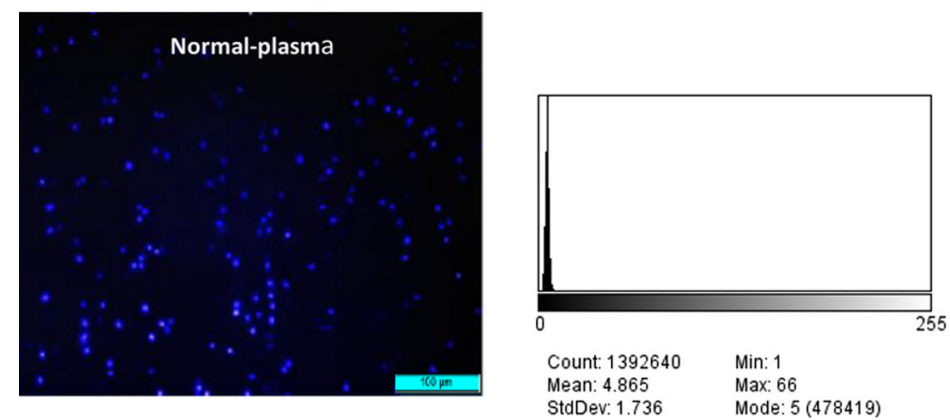

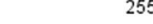

Min: 2

Max: 84
Mode: $11(1557187)$

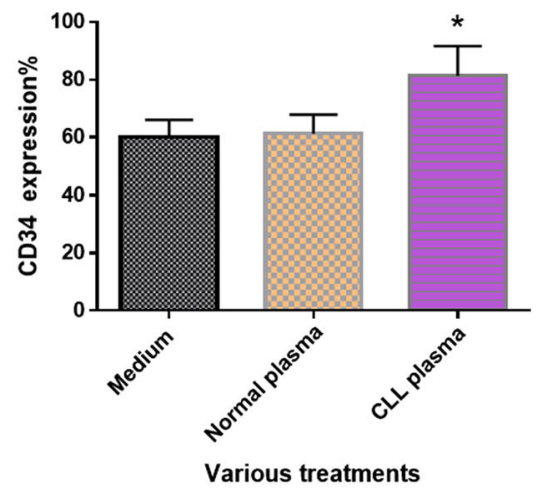

plasma. Green = FITC conjugate antibody. Left: Analysis of the collected images in different treatments via ImageJ software. Right: semiquantitative result of the images; data are presented as $\%$ mean $\pm \mathrm{SD}$
Fig. 5 Detection of CD34 using fluorescence microscopy. Serumstarved HUVECs were labeled with $\mathrm{mAb}$ against CD34. In the middle: representative illustrations of cells treated with CLL plasma and normal 

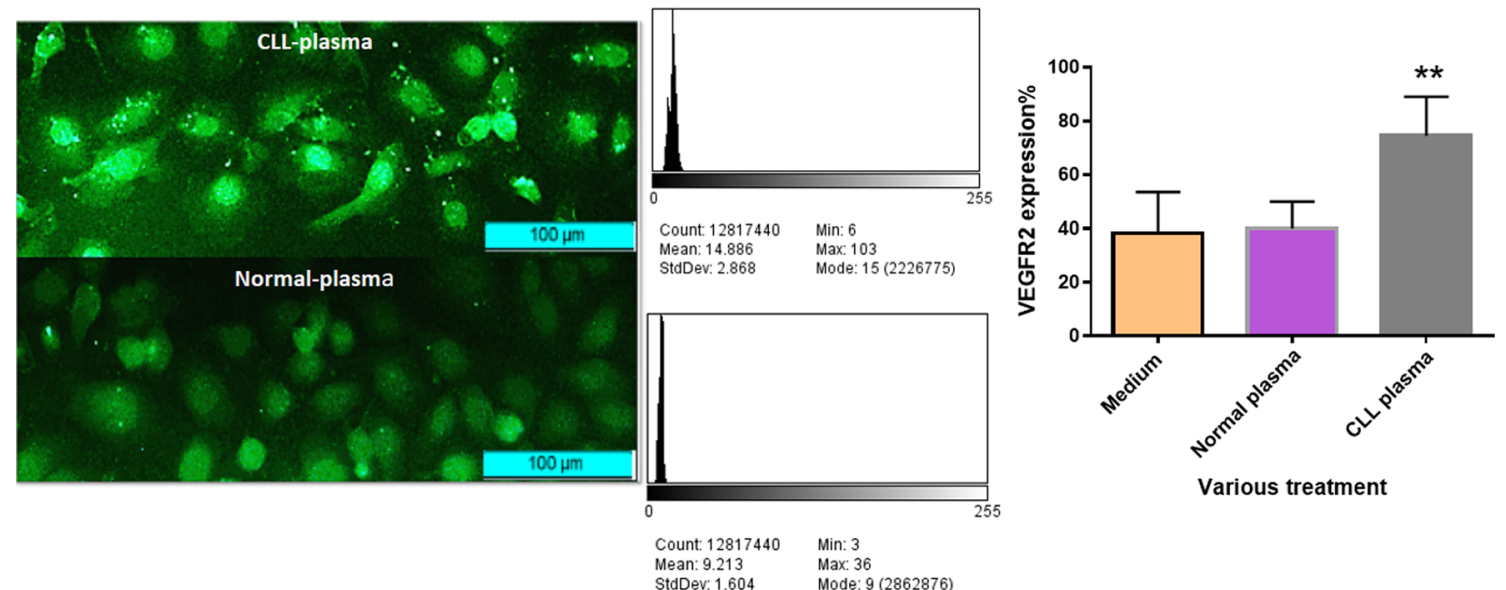

Fig. 6 Detection of VEGFR2 using fluorescence microscopy. Serumstarved HUVECs were labeled with man against VEGFR2. Left: representative illustrations of cells treated with CLL plasma and normal

plasma. Green = FITC conjugate antibody. Right: semi-quantitative evaluation of the collected images in different treatments via ImageJ software. Data are presented as $\%$ mean $\pm \mathrm{SD}$

inhibition, however, did not reverse the result significantly, when compared with siRNA control (data not shown).

Moreover, a greater effect on production of CD34-positive cells (up to $43 \%$ ) was observed upon treatment of the HUVECs with the plasma of the case with JAK2 mutation (Fig. 3).

Besides, a positive correlation between the number of CD34-positive cells and the absolute lymphocyte count was found $(P=0.0028, r=0.7132$; data not shown).

\section{Real-time quantitative PCR analysis}

To confirm the immunophenotype of the cells, the expression of CD34 mRNA by HUVECs was determined using real-time PCR. The cells were harvested over $24-48 \mathrm{~h}$ of incubation with CLL and normal plasmas. Complementary DNA (cDNA) synthesis and PCR were performed as described before, and the expression of the genes was normalized to the housekeeping gene subsequently. Incubation with CLL plasma increased the expression of CD34 and VEGFR2 mRNA after $48 \mathrm{~h}$, which was statistically significant, when compared with normal plasma ( $p=0.01$ and 0.0067 , respectively, Fig. 4).

\section{Immunocytochemistry}

To visualize CD34 and VEGFR2 expression in cell culture, immunocytochemistry experiments were carried out. The results of immunocytochemistry (antibody to CD34, Abcam) were analyzed via ImageJ software. A predominant CD34 expression was observed in the endothelial cells treated with CLL plasma when compared to the normal plasma (Fig. 5, $p=0.0004)$.

Moreover, the findings of VEGFR2 expression were analyzed via ImageJ software. A more predominant VEGFR2 expression, however, was observed in the CLL plasmatreated endothelial cells, when compared to the medium alone and normal plasma (Fig. 6, $p=0.0026$ and $p=0.0056$, respectively).

\section{Discussion}

Our experiments showed that CD34- and VEGFR2-positive human umbilical vein endothelial cells were increased dramatically following treatment with CLL plasma, particularly in cases with a higher absolute lymphocyte count after $48 \mathrm{~h}$ as detected by flow cytometry and immunocytochemistry. The amplified levels of CD34 and VEGFR2 mRNA using realtime PCR confirmed the above results. NF-kB inhibition, nevertheless, did not reverse the result significantly, when compared with siRNA control, indicating that the NF-kB signaling pathway plays no key role in this effect. A morphological alteration, however, was found in microscopic study, showing a number of the cells with extended filopodia. Our data indicate a further rationalization of the plasma angiogenic activities in CLL and confirm the result of the previous studies signifying that CLL could affect the angiogenic process via growth factor content in plasma [12-14]. However, this effect was powerfully amplified by the presence of JAK2 mutation in our study. Recently, a few groups reported JAK2 mutation in patients with CLL. This mutation has also been reported in a CLL patient coexisting with myeloproliferative neoplasm [15]. In addition, another group reported two CLL JAK2-positive cases and hypothesized that through the pre-JAK2 stage of the stem cell development, an early genetic hit may cause further mutations in these cells. These stem cells, however, may be able to differentiate into both myeloid and lymphoid lineages [16]. Though, JAK2 mutation was positively associated with VEGF and microvascular density. In addition, JAK2/STAT3 
signaling pathway was found to be linked with angiogenesis [17]. JAK2 inhibition, therefore, was introduced as a new pharmacological approach to CLL treatment $[18,19]$. The above data may indicate that CLL plasma as a potential source of VEGF influences on tip cell production resulting in exaggerated angiogenesis activity in CLL $[12,20]$.

Having just one CLL case with a JAK2 mutation and the lack of long-term follow-up have been the limitations of the present study. Coexistence of JAK 2 mutation with CLL, however, may potentiate angiogenesis. The results of our study, though, were consistent with our previous work, demonstrating a proliferative effect of the CLL plasma on endothelial cells [7]. Nevertheless, our preliminary data may introduce CLL plasma as a rational therapeutic target, particularly in cases with higher absolute lymphocyte count and/or JAK2 mutation. This may occur with increasing levels of angiogenic factors including VEGF and through the JAK2/STAT3 signaling pathway. Although there is much remains to be done, the present study might represent a new field of research in CLL development.

\section{Conclusions}

Taken together, the present study demonstrated that CLL plasma could prompt endothelial cell alteration to CD34-positive cells in human endothelial cells along with greater expression of VEGFR2 in these cells. Therefore, the data may recommend an angiogenic effect of CLL plasma. This may introduce CLL plasma as a candidate therapeutic target. Further investigations along with follow up studies, however, are needed to clarify the certain mechanism of the effects.

Acknowledgements We would like to thank the Research and Enterprise Committee and the School of Allied Medicine, Iran University of Medical Science, for their sustenance.

\section{Compliance with ethical standards}

Ethics statement Thestudywasapproved by theMedicalEthicsCommittee of Iran University of Medical Sciences (IR.IUMS.REC.1395.25330). Written informed consent was also obtained from all cases and normal subjects.

Conflict of interest The authors declare that they have no conflict of interest.

\section{References}

1. Gerhardt $\mathrm{H}$ et al (2003) VEGF guides angiogenic sprouting utilizing endothelial tip cell filopodia. J Cell Biol 161(6):1163-1177
2. Suchting $\mathrm{S}$ et al (2007) The notch ligand Delta-like 4 negatively regulates endothelial tip cell formation and vessel branching. Proc Natl Acad Sci 104(9):3225-3230

3. Bridges EM, Harris AL (2011) The angiogenic process as a therapeutic target in cancer. Biochem Pharmacol 81(10):1183-1191

4. Siemerink MJ et al (2013) Endothelial tip cells in ocular angiogenesis potential target for anti-angiogenesis therapy. J Histochem Cytochem 61(2):101-115

5. Nakatsu MN et al (2003) Angiogenic sprouting and capillary lumen formation modeled by human umbilical vein endothelial cells (HUVEC) in fibrin gels: the role of fibroblasts and Angiopoietin1 2 . Microvasc Res 66(2):102-112

6. Phng L-K, Stanchi F, Gerhardt H (2013) Filopodia are dispensable for endothelial tip cell guidance. Development 140(19):4031-4040

7. Jakobsson L et al (2010) Endothelial cells dynamically compete for the tip cell position during angiogenic sprouting. Nat Cell Biol 12(10):943-953

8. Chen $\mathrm{H}$ et al (2000) In vitro and in vivo production of vascular endothelial growth factor by chronic lymphocytic leukemia cells. Blood 96(9):3181-3187

9. ten Hacken E, Burger JA (2016) Microenvironment interactions and B-cell receptor signaling in chronic lymphocytic leukemia: implications for disease pathogenesis and treatment. Biochim et Biophys Acta (BBA)-Mol Cell Res 1863(3):401-413

10. Shahidi M, Razavi SM, Hayat P (2016) Induction of endothelial cell proliferation and von Willebrand factor expression and secretion by leukemic plasma of patients with chronic lymphocytic leukemia before and after inhibition of NF-[kappa] B. Blood Coagul Fibrinolysis

11. Shahidi $\mathrm{M}$ et al (2014) The in vitro effects of sodium salicylate on von Willebrand factor and C-reactive protein production by endothelial cells. Inflammopharmacology 22(6):367-372

12. Gora-Tybor J, Blonski JZ, Robak T (2005) Circulating vascular endothelial growth factor (VEGF) and its soluble receptors in patients with chronic lymphocytic leukemia. Eur Cytokine Netw 16(1):41-46

13. Molica S et al (2002) Clinicoprognostic implications of increased serum levels of vascular endothelial growth factor and basic fibroblastic growth factor in early B-cell chronic lymphocytic leukaemia. Br J Cancer 86(1):31-35

14. Xia Y, Lu R-N, Li J (2012) Angiogenic factors in chronic lymphocytic leukemia. Leuk Res 36(10):1211-1217

15. Kodali $\mathrm{S}$ et al (2009) JAK2 mutation in a patient with CLL with coexistent myeloproliferative neoplasm (MPN). Leuk Res 33(12): e236-e239

16. Tabaczewski P, Nadesan S, Lim SH (2009) Zap-70 positive chronic lymphocytic leukemia co-existing with Jak 2 V671F positive essential thrombocythemia: a common defective stem cell? Leuk Res 33(6):854-855

17. Lu YM et al (2016) Eriocalyxin B blocks human SW1116 colon cancer cell proliferation, migration, invasion, cell cycle progression and angiogenesis via the JAK2/STAT3 signaling pathway. Mol Med Rep 13(3):2235-2240

18. Montresor A et al (2015) JAK2 tyrosine kinase mediates integrin activation induced by CXCL12 in B-cell chronic lymphocytic leukemia. Oncotarget 6(33):34245

19. Abramson HN (2016) Kinase inhibitors as potential agents in the treatment of multiple myeloma. Oncotarget 7(49):81926-81968

20. Maffei $\mathrm{R}$ et al (2010) Increased angiogenesis induced by chronic lymphocytic leukemia B cells is mediated by leukemia-derived Ang2 and VEGF. Leuk Res 34(3):312-321 